\title{
A Method for Analyzing Correlation Grades of Factors Influencing the Behavior of Bolted Connections Based on the Grey Correlation Degree
}

\author{
Qishui YAO, Qiuwei YUAN, Jianghong YU*, Yaoyao DENG
}

\begin{abstract}
Failure caused by loosening of bolted connections is a common problem in practice. To reasonably optimize the factors influencing the behavior of bolted connections, this paper proposed a method for analyzing the correlation grades of factors influencing the behavior of bolted connection based on the grey correlation degree. First, Deng's correlation degree is optimized from three different perspectives: dimensionless factors, resolution coefficient, and weight assignment. Then, a model of the bolted connection is established based on the degrees of correlation of influencing factors. To avoid inverse correlation of influencing factors caused by fluctuation of the residual preload, a difference classification method based on qualitative analysis is proposed. By grading the correlation degree of each influencing factor, optimal selection of parameters in bolted connections can be achieved. Finally, the bolted connection on a third-rail current collector slide was taken as an example and residual preload analysis was performed. It can be concluded that reasonably selecting the bolt material and size and optimizing the friction condition of various contact surfaces is necessary to effectively improve the reliability of the bolted connection. The method was verified by simulations.
\end{abstract}

Keywords: bolt connection; correlation grade analysis method; difference classification method; grey correlation degree; third-rail current collector slide

\section{INTRODUCTION}

Bolted connections, as common fasteners, are widely used, for example, in trains, buildings, and aircraft, mainly owing to their easy mounting, convenient assembly, and reliable performance $[1,2]$. However, bolted connections are often exposed to vibrations throughout their service life, and are thus susceptible to loosening, fracture, and other forms of failure. For this reason, factors influencing the behavior of bolted connections should be optimized to improve their reliability.

Scholars both at home and abroad have explored the relationship between influencing factors and reliability using three different approaches: theoretical analysis, simulation, and experiments. In terms of theory, Hou [3] established a one-dimensional (1D) mathematical model of a bolt that considers elastoplasticity and creep effects, and analyzed the variation in stress, strain, and tension in the threads with temperature. Vingradov and Huang [4] introduced a dynamic model of a bolted connection and showed that high-frequency vibrations can lead to loosening of the bolted connections.

In the area of simulations, Yang [5] built a finite element model of a bolted connection with a metal housing and comparatively analyzed the stresses in bolts with and without a bolt fixing structure. The presence of a bolt fixing structure resulted in a more reasonable stress distribution in the bolt. Ren [6] developed a finite element model of the propulsion shafting system of a container ship and investigated the relationship between the number of bolts and shafting vibration. The results suggest resonance amplitude, assembly/disassembly, and other practical issues should be comprehensively considered in selecting the appropriate number of bolts. Wang [7] developed a finite element model of a bolt under lateral excitation and analyzed the effects of initial preload and coefficient of friction on the loosening mechanism of the bolt. Yang [8] introduced a parametric three-dimensional (3D) finite element model for bolted connections and analyzed the mechanical properties of connections containing bolts of various pitches and different numbers of threads.

On the experimental front, Junker [9] introduced the
Junker looseness tester and found that compared to an axial load of the same magnitude, the dynamic lateral load more easily leads to loosening of bolted connections [10]. Yang tested the double-nut bolts used in transmission towers under lateral vibration and showed that an ordinary doublenut bolted connection is more reliable when the mounting torque of the lower nut is $25 \%$ of the upper nut mounting torque.

Based on the above review of the literature, to date, studies have only focused on the influence of relevant factors on the reliability of bolted connections, whereas the degree of correlation of multiple influencing factors and the reliability of bolted connections have not been considered. This paper presents a method for analyzing the correlation grades of factors influencing the behavior of bolted connection based on grey system theory. Factors influencing the reliability of bolted connections can be classified and graded, which not only allows inversion of the correlation order of influencing factors caused by fluctuation of the residual preload to be avoided, but also optimizes the influencing factors in a targeted manner. First, on the basis of Deng's correlation degree, optimization is performed from three perspectives, that is, dimensionless processing of factors, resolution coefficients, and weight assignments. Then, the difference classification method based on qualitative analysis is introduced and combined with grey correlation degree analysis to develop a method for analyzing the correlation grade factors influencing the behavior of bolted connections, which can be used in qualitative analyses. Finally, to verify the proposed method, correlation grades of factors influencing the behavior of bolted connections on a third-rail current collector slide were analyzed.

\section{GREY CORRELATION DEGREE}

Grey system theory [11] does not require massive experimental datasets or prior probability distributions and has therefore become widely used in the fields of management and engineering [12, 13]. However, grey correlation analysis is rarely applied to analyze mechanical parts, especially bolted connections. The grey correlation 
degree is an essential component of grey system theory and reflects the degree of correlation between an event and an influencing factor. The judgment is based on the similarity degree between the curve of the event data sequence and the curve of the data sequence of the influencing factor. A higher similarity degree between two curves suggests a higher correlation between the event and influencing factor; and vice versa.

Depending on the structure and mechanism, correlation degrees can be classified as follows: Deng's correlation degree, absolute correlation degree, relative correlation degree, slope correlation degree, T-type correlation degree, B-type correlation degree, C-type correlation degree, etc.

In this paper, the grey correlation degree, based on both Deng's correlation degree and the relative correlation degree, is introduced.

The data sequence of an influencing factor can be defined as where $i$ denotes the number of influencing factors, $i=1,2, \ldots, n ; k$ is the index number; $x_{i}(k)$ is the observed data of influencing factor $X_{i}$ relative to the $k^{\text {th }}$ index.

The event data sequence can be defined as

$X_{i}=\left(x_{i}(1), x_{i}(2), \cdots, x_{i}(k), \cdots, x_{i}(n)\right)$

where $y_{j}(k)$ is the observed data of data sequence $Y_{j}$ relative to the $k^{\text {th }}$ index.

$Y_{j}=\left(y_{j}(1), y_{j}(2), \cdots, y_{j}(k), \cdots, y_{j}(n)\right)$

\subsection{Deng's Correlation Degree}

An image conversion process is first performed to reduce the effects of differences among various data indices, in terms of both dimension and order, on the correlation degree calculation.

Assuming $D$ is the sequential algorithm, then where $x_{i}(k) \cdot d=x_{i}(k) / x_{i}(1) \neq 0$

$$
X_{i}^{\prime}=X_{i} \cdot D=\left(x_{i}(1) \cdot d, x_{i}(2) \cdot d, \cdots, x_{i}(k) \cdot d, \cdots, x_{i}(n) \cdot d\right)
$$

In this case, $D$ is the initial-value operator and $X_{i}^{\prime}$ is the image of $X_{i}$ under $D$ (referred to as the initial-value image).

Data sequences $X_{i}$ and $Y_{j}$ undergo initial-value image conversion.

The difference sequence is defined as where $\Delta_{i j}=$ $\left(\Delta_{i j}(1), \Delta_{i j}(2), \ldots, \Delta_{i j}(n)\right)$

$$
\Delta_{i j}(k)=\left|x_{i}{ }^{\prime}(k)-y_{j}^{\prime}(k)\right|
$$

The maximum absolute difference and minimum absolute difference are defined as

The correlation coefficients of data sequences $X_{i}$ and $Y_{j}$ are

$$
\begin{aligned}
& M_{j}=\max \Delta_{i j}(k), m_{j}=\min \Delta i_{j}(k) \\
& \xi_{i j}(k)=\frac{m_{j}+\xi M_{j}}{\Delta i j+\xi M_{j}}, \xi \in(0,1]
\end{aligned}
$$

where $\xi$ is the resolution coefficient, which can change the difference between correlation coefficients, and is normally 0.5 .

In this case, the initial-value correlation degree of data sequences $X_{i}$ and $Y_{j}$ is

\subsection{Relative Correlation Degree}

Initial-value images $X_{i}^{\prime}$ and $Y_{j}^{\prime}$ of $X_{i}$ and $Y_{j}$ can be obtained from Section 1.1. Assuming

$\gamma_{i j}=\frac{1}{n} \sum_{k=1}^{n} \xi_{i j}(k), i=1,2, \cdots m$

The relative correlation degree becomes

$$
\begin{aligned}
& \left|S_{i}\right|=\left|\sum_{2}^{n-1} X_{i}^{\prime}(k)+\frac{1}{2} X_{i}^{\prime}(n)\right| \\
& \left|S_{j}\right|=\left|\sum_{2}^{n-1} Y_{j}^{\prime}(k)+\frac{1}{2} Y_{j}^{\prime}(n)\right| \\
& \left|S_{i}-S_{j}\right|=\left|\sum_{2}^{n-1}\left[X_{i}^{\prime}(k)-Y_{j}^{\prime}(k)\right]+\frac{1}{2} Y_{j}^{\prime}(n)-\frac{1}{2} X_{i}^{\prime}(n)\right|
\end{aligned}
$$

\subsection{Problems with Traditional Correlation Degrees}

$$
\gamma_{i j}=\frac{1+\left|S_{i}\right|+\left|S_{j}\right|}{1+\left|S_{i}\right|+\left|S_{j}\right|+\left|S_{i}-S_{j}\right|}
$$

1) The resolution coefficient $\xi$ in Deng's correlation degree affects the magnitude of the correlation degree and influences the correlation degrees of the influencing factors. The relative correlation degree calculation does not cover resolution coefficients and lacks judgment about the overall trend, and is therefore one-sided.

2) Traditional correlation degrees have not taken into account differences in the influence of various factors on the event. Different factors will have different weights and neglecting these weights can lead to significant deviation from reality.

\section{METHOD FOR ANALYZING THE CORRELATION GRADES OF BOLTED CONNECTIONS}

To address the difficulties in optimally selecting the various factors influencing the behavior of bolted connections, dimensionless processing of factors is introduced as the optimization method based on Deng's correlation degree. First, mean-value images of various data sequences are obtained. Then, coefficients with suitable resolutions are defined to acquire the correlation coefficients. Next, the objective weighting method and information entropy principle are used to assign weights to the influencing factors and the correlation degrees are calculated. Finally, the difference classification method is used to grade each correlation degree and commonalities among factors of the same grade are summarized. As such, 
the correlation degree between the reliability of the bolted connection and relevant influencing factors can be obtained according to their specific correlation grades, with the overall aim of identifying the optimal scheme.

\subsection{Dimensionless Data Processing}

Differences exist among factors influencing bolted connections in terms of both dimension and order of magnitude; therefore, dimensionless processing is necessary. Traditional correlation degrees can achieve initial-value processing of sequence data. However, data sequences of the influencing factors of bolted connections are random and without any definite time order, thus, data processing is required in this case. The mean value method is adopted and can be described as follows:

Where $X_{i}^{\prime}$ is the image of $X_{i}$ under the mean-value operator, referred to as the mean-value image.

$X_{i}^{\prime}=X_{i} \cdot D=\left(x_{i}(1) \cdot d, x_{i}(2) \cdot d, \ldots, x_{i}(k) \cdot d, \ldots, x_{i}(n) \cdot d\right)$

Where

$$
x_{i}(k) \cdot d=\frac{x_{i}(k)}{\frac{i}{n} \sum_{k=1}^{n} x_{i}(k)}
$$

\subsection{Selection of Resolution Coefficients}

In determining correlation degrees influencing factors of bolted connections, the magnitude of the resolution coefficient will have some effect on the results. According to Eq. (6), the product of resolution coefficient $\xi$ and the maximum absolute difference have a significant effect on the correlation coefficients. To ensure the results are more aligned with reality, resolution coefficient $\xi$ should meet the following basic criteria:

When sequence data values are stationary, the selected $\xi$ value should enhance their discriminability, thereby embodying integrity.

2) When singular values exist among sequence data values, the selected $\xi$ value should weaken the effects of singular values on correlation coefficients.

To meet these conditions, the following values should be assigned to resolution coefficient $\xi$ :

1) When $M \geq \frac{3}{N} \sum_{i=1}^{N}\left|x_{i}^{\prime}(k)-y^{\prime}(k)\right|$, singular values exist among sequence data values. In this case, a small value should be assigned to resolution coefficient $\xi$ within the interval $\left[\theta_{i}, 1.5 \theta_{i}\right]$, thus weakening the effect of $M$ on the correlation coefficients. Normally, the right endpoint of the interval is selected according to the monotonicity of the function, i.e., $1.5 \theta_{i}$.

2) When $M \leq \frac{3}{N} \sum_{i=1}^{N}\left|x_{i}^{\prime}(k)-y^{\prime}(k)\right|$, the sequence data values are stationary. In this case, a large value should be assigned to resolution coefficient $\xi$ within the interval $\left[1.5 \theta_{i}, 2 \theta_{i}\right]$, thus enhancing the effect of $M$ on the correlation coefficients, which is normally set to $2 \theta_{i}$, where

$$
\theta_{i}=\frac{3 \sum_{i=1}^{N}\left|x_{i}^{\prime}(k)-y^{\prime}(k)\right|}{M \cdot N} .
$$

\subsection{Determination of Weights of Influencing Factors}

In the analysis of correlation degrees of bolted connections, different influencing factors will have different weights. Assigning the same weight values would produce deviations in the correlation degree calculation. The weights assigned to different influencing factors should reflect their different roles. The main methods for determining the weights of influencing factors include the subjective weighting method and the objective weighting method. Considering the small effects of subjective factors on the factors influencing the behavior of bolted connections, the objective weighting method is adopted. More specifically, the entropy weight method is used to assign weight, thus overcoming the problems of traditional approaches to mean correlation degrees calculations. The specific steps of the calculation are as follows:

1) Calculate feature weight $p_{i k}$ of the $i^{\text {th }}$ influencing factor in the $k^{\text {th }}$ bolted connection model:

$p_{i k}=\frac{x_{i}^{\prime}(k)}{\sum_{i=1}^{m} x_{i}^{\prime}(k)}$

2) Calculate entropy value $h_{k}$ of the $k^{\text {th }}$ bolted connection model:

$h_{k}=-\frac{1}{\ln m} \sum_{i=1}^{m} p_{i k} \cdot \ln p_{i k}$

3) Calculate weight value $\omega_{k}$ of the $k^{\text {th }}$ bolted connection model:

$$
\omega_{k}=\frac{1-h_{k}}{n-\sum_{k=1}^{n} h_{k}}
$$

4) Calculate the improved grey correlation degree:

$$
\gamma_{i j}=\sum_{i=1}^{n} \xi_{i j}(k) \omega_{k}
$$

\subsection{Grading of Correlation Degree Based on Difference Classification}

Bolted connections must remain stable under vibrations, however, their behavioral data sequences tend to fluctuate to some extent. For instance, a bolted connection in a stable state will have different residual preloads at different time points, which induce fluctuation in the correlation degrees and may cause inversion of the correlation matrix and compromise the accuracy of the results. To attain consistent conclusions, it is necessary to comparatively analyze correlation degrees by grading them based on difference classification. 
The difference classification method grades correlation degrees according to their differences. A correlation difference $(m)$ is introduced based on the differences between the overall correlation degrees. Influencing factors with the highest correlation degree are automatically classified as grade I; adjacent factors with a difference of less than $m$ are classified into the same grade; a factor presenting a difference of greater than $m$ from its adjacent factor is classified into the next grade. Thus, by parity of reasoning, influencing factors are classified into three correlation grades. A common solution is sought for all influencing factors within the same correlation grade.

\section{EXAMPLE ANALYSIS}

In this section, the correlation grades of influencing factors of the bolted connections on a third-rail current collector slide are analyzed to verify the proposed method.

\subsection{Building of Third-Rail Current Collector Model}

A third-rail current collector model and bolted connection model were established for the analysis. The third-rail current collector system is comprised of a fuse box, swing arms, current collector slides, and various other parts. A 3D model was built in CREO using the actual parameters of the system, then imported into ABAQUS for finite element analysis. The finite element model is shown in Fig. 1.

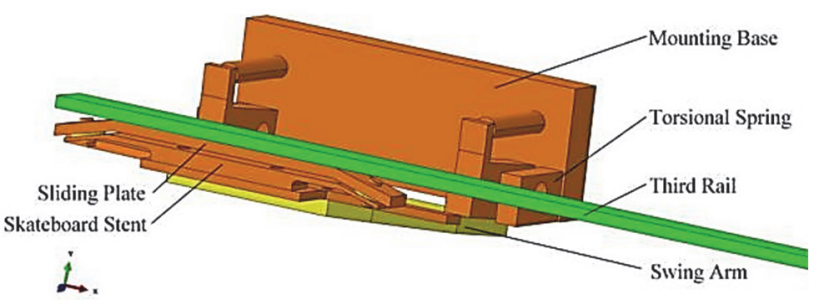

Figure 1 Finite element model of third-rail current collector

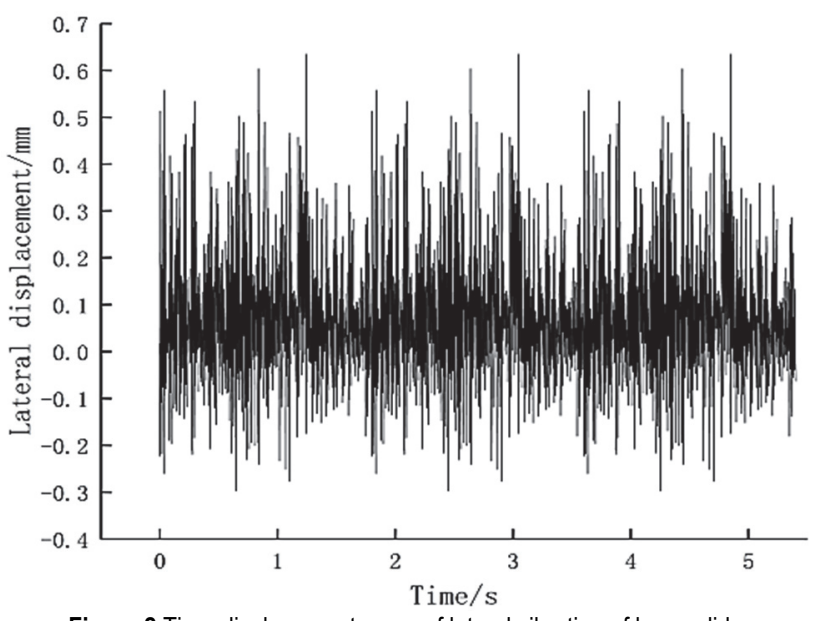

Figure 2 Time-displacement curve of lateral vibration of lower slide

During operation, trains are exposed to the random vibrations caused by track irregularities. In this study, the fifth grade American track spectrum was converted through time-frequency transformation into a timedisplacement curve in MATLAB, and applied as the vibration excitation to the finite element model. A speed of $60 \mathrm{~km} / \mathrm{h}$, which is the optimal running speed of a third-rail train, was also adopted for the simulations and a lateral vibration time-displacement curve of the lower slide was obtained, as shown in Fig. 2.

The simulated lateral vibration excitation was adopted as the vibration excitation for precise modeling of the bolted connection.

\subsection{Bolted Connection Model}

Modeling of the bolted connection was carried out using the actual parameters of slide bolts. The model contained the lead angle, and was capable of precisely simulating the change in residual preload in the bolted connection. The bolts were distributed symmetrically on both sides of the slides. Only one bolt was analyzed. The slider shape was simplified and contact surfaces for applying the lateral vibration displacement were retained. Fig. 3 shows the precise, simplified finite element model of the bolted connection.

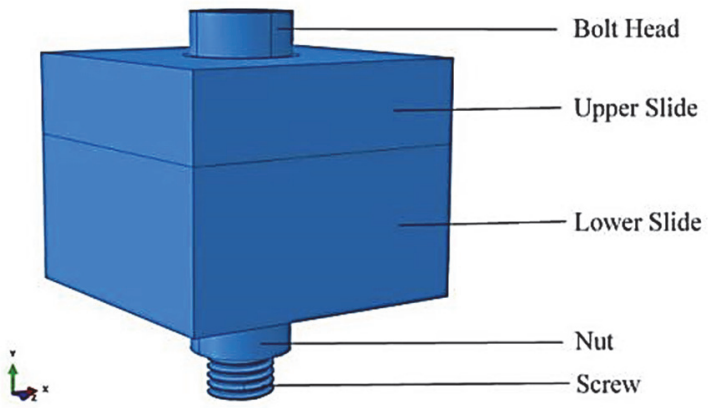

Figure 3 Finite element model of bolted connection

Contact pairs were arranged on the bolt head pressurebearing surface, nut pressure-bearing surface, sliding contact surface, and thread engagement surface. A preload element was applied in the middle of the bolt bar, and its length was fixed to sustain the preload effect. Based on the actual operating conditions, the upper surface of the upper slide was fully constrained, the two sides, parallel to plane $Y O Z$ were constrained in the $X$-direction, and the two sides parallel to plane $Y O X$ were constrained in the $Z$-direction. On the lower slide, the two sides parallel to plane $Y O Z$ were constrained in the $X$-direction.

The two sides of the lower slide parallel to plane $Y O Z$ were constrained in the $X$ direction. The sides of the lower slide parallel to plane $Y O X$ were applied with the vibration load input extracted in Section 4.1 in the Z-direction, followed by the finite element analysis and calculations.

\subsection{Data Analysis}

The results of the simulation model were used as data for further analysis. The 12 influencing factors of the bolted connection model and the residual preload results of the simulation analysis were taken as the study objects. For the convenience of analysis, the symbols and meanings used are shown in Tab. 1. Data values of influencing factors of 12 different bolted connection models are shown in Tab. 2. At time t, the residual preload is $Y_{j}, j=1,2,3,4$, 5,6 . The values of $Y_{j}(k)$ correspond to the residual preload values of the $k^{\text {th }}$ bolted connection at $1 \mathrm{~s}, 1.2 \mathrm{~s}, 1.4 \mathrm{~s}, 1.6 \mathrm{~s}$, $1.8 \mathrm{~s}$, and $2 \mathrm{~s}$, given in Tab. 3. 
Table 1 Definition of symbols used in finite element model of bolted connection

\begin{tabular}{|c|l|}
\hline \multicolumn{1}{|c|}{ Table 1 Definition of symbols used in finite element model of bolted connection } \\
\hline$X_{1}$ & Bolt nominal diameter \\
\hline$X_{2}$ & Bolt pitch \\
\hline$X_{3}$ & Screw length \\
\hline$X_{4}$ & Thread length \\
\hline$X_{5}$ & Bolt head height \\
\hline$X_{6}$ & Nut thickness \\
\hline$X_{7}$ & Bolt connection gap \\
\hline$X_{8}$ & Upper slide thickness \\
\hline$X_{9}$ & Lower slide thickness \\
\hline$X_{10}$ & Bolt and nut elastic modulus \\
\hline$X_{11}$ & Coefficient of friction of contact pair on thread engagement surface \\
\hline$X_{12}$ & Coefficient of friction of contact pair on bolt head and nut pressure-bearing surface \\
\hline$X_{13}$ & Coefficient of friction of contact pair on upper/lower slide pressure-bearing surface \\
\hline$Y_{\mathrm{j}}$ & Residual preload value at time $t$ \\
\hline
\end{tabular}

Table 2 Influencing factors values

\begin{tabular}{|c|c|c|c|c|c|c|c|c|c|c|c|c|c|c|}
\hline$k$ & $X_{1}$ & $X_{2}$ & $X_{3}$ & $X_{4}$ & $X_{5}$ & $X_{6}$ & $X_{7}$ & $X_{8}$ & $X_{9}$ & $X_{10}$ & $X_{11}$ & $X_{12}$ & $X_{13}$ \\
\hline 1 & 10 & 1.5 & 60 & 26 & 6.4 & 8.4 & 0.6 & 25.3 & 12.7 & 210 & 0.15 & 0.2 & 0.13 \\
\hline 2 & 12 & 1.75 & 65 & 30 & 7.5 & 10.8 & 0.4 & 16.8 & 23 & 200 & 0.1 & 0.18 & 0.22 \\
\hline 3 & 14 & 1.85 & 70 & 24 & 8.8 & 12.8 & 0.5 & 17 & 30.8 & 190 & 0.08 & 0.25 & 0.12 \\
\hline 4 & 16 & 2 & 78 & 29 & 10 & 14.8 & 0.3 & 27.2 & 26 & 220 & 0.22 & 0.17 & 0.11 \\
\hline 5 & 18 & 2.25 & 85 & 42 & 11.5 & 15.8 & 0.55 & 11.3 & 36.8 & 230 & 0.12 & 0.19 & 0.3 \\
\hline 6 & 20 & 2.5 & 95 & 46 & 12.5 & 18 & 0.9 & 34.9 & 26 & 240 & 0.29 & 0.14 & 0.23 \\
\hline 7 & 22 & 2.6 & 100 & 50 & 14 & 19.4 & 0.5 & 31.1 & 42.4 & 260 & 0.16 & 0.27 & 0.31 \\
\hline 8 & 24 & 2.8 & 105 & 54 & 15 & 21.5 & 0.7 & 35.3 & 17 & 170 & 0.09 & 0.23 & 0.15 \\
\hline
\end{tabular}

Table 3 Residual preload value $Y_{j}$ at time $t$

\begin{tabular}{|c|c|c|c|c|c|c|}
\hline Time $t$ & $Y_{1}$ & $Y_{2}$ & $Y_{3}$ & $Y_{4}$ & $Y_{5}$ & $Y_{6}$ \\
\hline 1 & 14717.8 & 14461.8 & 14281.8 & 14250.3 & 14257.6 & 13992.5 \\
\hline 2 & 19471.6 & 19426.8 & 19439.1 & 19436.2 & 19448.3 & 19400.5 \\
\hline 3 & 18088.8 & 17805.4 & 17734.7 & 17694.1 & 17659.8 & 17481.4 \\
\hline 4 & 19654.7 & 20452.5 & 19606.4 & 19627.3 & 19600.3 & 19282.1 \\
\hline 5 & 20070.4 & 20063.8 & 20114.6 & 20171.4 & 20126.9 & 20197.9 \\
\hline 6 & 19986.2 & 21490.7 & 19620.1 & 19598.6 & 19547.2 & 19341.3 \\
\hline 7 & 18639.2 & 18294.5 & 18136.7 & 18008.6 & 17858.2 & 17649.4 \\
\hline 8 & 19966.7 & 19977.1 & 19995.7 & 20005.9 & 19943 & 19972.1 \\
\hline
\end{tabular}

The mean value method was applied to the data sequences in Tab. 2 to obtain mean-value images, which were then used to solve the difference sequence, maximum absolute difference, and minimum absolute difference using Eq. (5). The judgment criteria presented in Section 3.2 were used to obtain the resolution coefficients of the sequence data. By determining the weights of the influencing factors, Eqs. (14) to (17) could then be used to solve for the weight values of the various bolt models and to acquire the correlation degrees between the various influencing factors and residual preload of the bolt at time $t$. The results are presented in Tab. 4 .

Table 4 Correlation degree between influencing factors of bolted connections and bolt residual preload at time $t$

\begin{tabular}{|c|c|c|c|c|c|c|c|c|c|c|c|c|}
\hline \multirow[b]{2}{*}{ No. } & \multicolumn{2}{|c|}{$1 \mathrm{~s}$} & \multicolumn{2}{|c|}{$1.2 \mathrm{~s}$} & \multicolumn{2}{|c|}{$1.4 \mathrm{~s}$} & \multicolumn{2}{|c|}{$1.6 \mathrm{~s}$} & \multicolumn{2}{|r|}{$1.8 \mathrm{~s}$} & \multicolumn{2}{|r|}{$2 \mathrm{~s}$} \\
\hline & Symbol & $\begin{array}{c}\text { Correlation } \\
\text { degree }\end{array}$ & Symbol & $\begin{array}{c}\text { Correlation } \\
\text { degree }\end{array}$ & Symbol & $\begin{array}{c}\text { Correlation } \\
\text { degree }\end{array}$ & Symbol & $\begin{array}{c}\text { Correlation } \\
\text { degree }\end{array}$ & Symbol & $\begin{array}{c}\text { Correlation } \\
\text { degree }\end{array}$ & Symbol & $\begin{array}{c}\text { Correlation } \\
\text { degree }\end{array}$ \\
\hline 1 & $X_{10}$ & 0.7466 & $X_{10}$ & 0.7335 & $X_{10}$ & 0.7439 & $X_{10}$ & 0.7517 & $X_{10}$ & 0.7513 & $X_{10}$ & 0.7793 \\
\hline 2 & $X_{3}$ & 0.7165 & $X_{3}$ & 0.7313 & $X_{3}$ & 0.7267 & $X_{3}$ & 0.7363 & $X_{3}$ & 0.7345 & $X_{3}$ & 0.7714 \\
\hline 3 & $X_{2}$ & 0.7033 & $X_{2}$ & 0.7172 & $X_{2}$ & 0.7119 & $X_{2}$ & 0.7211 & $X_{2}$ & 0.7195 & $X_{2}$ & 0.7541 \\
\hline 4 & $X_{5}$ & 0.6492 & $X_{4}$ & 0.6553 & $X_{5}$ & 0.6614 & $X_{5}$ & 0.6692 & $X_{5}$ & 0.6682 & $X_{5}$ & 0.6974 \\
\hline 5 & $X_{1}$ & 0.6474 & $X_{1}$ & 0.6517 & $X_{1}$ & 0.6516 & $X_{4}$ & 0.6596 & $X_{4}$ & 0.6585 & $X_{4}$ & 0.6887 \\
\hline 6 & $X_{6}$ & 0.6465 & $X_{6}$ & 0.6504 & $X_{4}$ & 0.6516 & $X_{1}$ & 0.6594 & $X_{1}$ & 0.6584 & $X_{6}$ & 0.6885 \\
\hline 7 & $X_{4}$ & 0.6463 & $X_{5}$ & 0.6463 & $X_{6}$ & 0.6510 & $X_{6}$ & 0.6590 & $X_{6}$ & 0.6579 & $X_{1}$ & 0.6879 \\
\hline 8 & $X_{12}$ & 0.5891 & $X_{7}$ & 0.5727 & $X_{12}$ & 0.5948 & $X_{12}$ & 0.6026 & $X_{12}$ & 0.6024 & $X_{12}$ & 0.6315 \\
\hline 9 & $X_{7}$ & 0.5752 & $X_{12}$ & 0.5500 & $X_{7}$ & 0.5855 & $X_{7}$ & 0.5946 & $X_{7}$ & 0.5955 & $X_{7}$ & 0.6224 \\
\hline 10 & $X_{9}$ & 0.5446 & $X_{13}$ & 0.5005 & $X_{9}$ & 0.5520 & $X_{9}$ & 0.5599 & $X_{9}$ & 0.5597 & $X_{9}$ & 0.5902 \\
\hline 11 & $X_{13}$ & 0.4964 & $X_{9}$ & 0.4968 & $X_{13}$ & 0.5078 & $X_{13}$ & 0.5160 & $X_{13}$ & 0.5160 & $X_{13}$ & 0.5431 \\
\hline 12 & $X_{8}$ & 0.4940 & $X_{8}$ & 0.4938 & $X_{8}$ & 0.5042 & $X_{8}$ & 0.5125 & $X_{8}$ & 0.5130 & $X_{8}$ & 0.5320 \\
\hline 13 & $X_{11}$ & 0.4286 & $X_{11}$ & 0.4104 & $X_{11}$ & 0.4296 & $X_{11}$ & 0.4348 & $X_{11}$ & 0.4348 & $X_{11}$ & 0.4498 \\
\hline
\end{tabular}

\subsection{Grading of Correlation Degree Based on Difference Classification}

According to the order of six groups of correlation degrees in Tab. 4, when correlation difference $m$ is $5 \%$, the influencing factors are clearly graded. Thus, correlation difference $m$ was set as $5 \%$ for the difference classification of correlation degree. Tab. 5 presents the correlation grades of the influencing factors.

Table 5 Correlation grades of influencing factors \begin{tabular}{l|l} 
Correlation grade & Influencing factor \\
\hline
\end{tabular}

\begin{tabular}{|l|c|}
\hline First correlation grade & $X_{10}, X_{3}, X_{2}$ \\
\hline Second correlation grade & $X_{5}, X_{1}, X_{6}, X_{4}$ \\
\hline Third correlation grade & $X_{12}, X_{7}, X_{9}, X_{8}, X_{13}, X_{11}$ \\
\hline
\end{tabular}

From Tab. 5, the first correlation grade contains the 
elastic modulus of the bolt, screw length, and bolt pitch. Elastic modulus is a measure of the bolt's resistance to elastic deformation and a basic material property; therefore, it is important to select a suitable bolt material. Screw length is related to the thickness of the connected part, and should be minimized under the premise of maintaining reliability of the bolted connection.

The second correlation grade contained bolt head height, bolt nominal diameter, nut thickness, and thread length, all of which are basic parameters of standard bolts. Therefore, to strengthen the reliability of the bolted connection, choosing a suitable bolt is vital.

The third correlation grade covers the coefficient of friction of the contact pair on the bolt head and nut pressure-bearing surface, bolt connection gap, lower slide thickness, coefficient of friction of the contact pair on the upper/lower slide pressure-bearing surface, upper slide thickness, and coefficient of friction of the contact pair on the thread engagement surface. All of these are environmental factors related to both the operating state and operating environment.

Analysis of the above three correlation grades reveals that the key to strengthening the reliability of bolted connections lies in selecting a suitable bolt material and size. After a suitable bolt size is selected, the next step is to properly adjust the friction conditions of the contact surfaces.

\subsection{Simulation Verification}

The correlation degrees of the bolted connection were verified using the control variable method. A bolted connection model was established according to the actual size of the M14 bolts on a third-rail current collector slide. Bolt sizes are specified in national standards and cannot be arbitrarily modified. Thus, for the bolt connection model, the elastic modulus of the bolt material $X_{10}$, coefficient of friction of the contact pair on the upper/lower slide pressure-bearing surface $X_{13}$, coefficient of friction of the contact pair on the thread engagement surface $X_{11}$, and coefficient of friction of the contact pair on the bolt head and nut pressure-bearing surface $X_{12}$ were varied by $10 \%$ while all other parameters remained unchanged in order to compare the magnitude of their effects on the residual preload. Fig. 4 shows the changes in the ratio of residual preload to initial preload with vibration time as certain parameters are varied.

From Fig. 4, it can be observed that when vibration time is in the range of $1 \mathrm{~s}$ to $2 \mathrm{~s}$, the residual preload ratios can be ranked in descending order as follows: $X_{10}>X_{12}>$ $X_{13}>X_{11}$. The correlation order varies with time, however, as shown in Tab. 4, in the order of six groups of correlation degrees under analysis, influencing factors $X_{10}, X_{11}, X_{12}$, and $X_{13}$ present the same order as $X_{10}, X_{12}, X_{13}$ and $X_{11}$ in descending order of correlation degree. This is consistent with the conclusions drawn from Fig. 4, and verifies the reliability of the method for analyzing the degree correlation between influencing factors and behavior of bolted connections. In addition, reasonableness of the method for analyzing the correlation grades of bolted connections was confirmed.

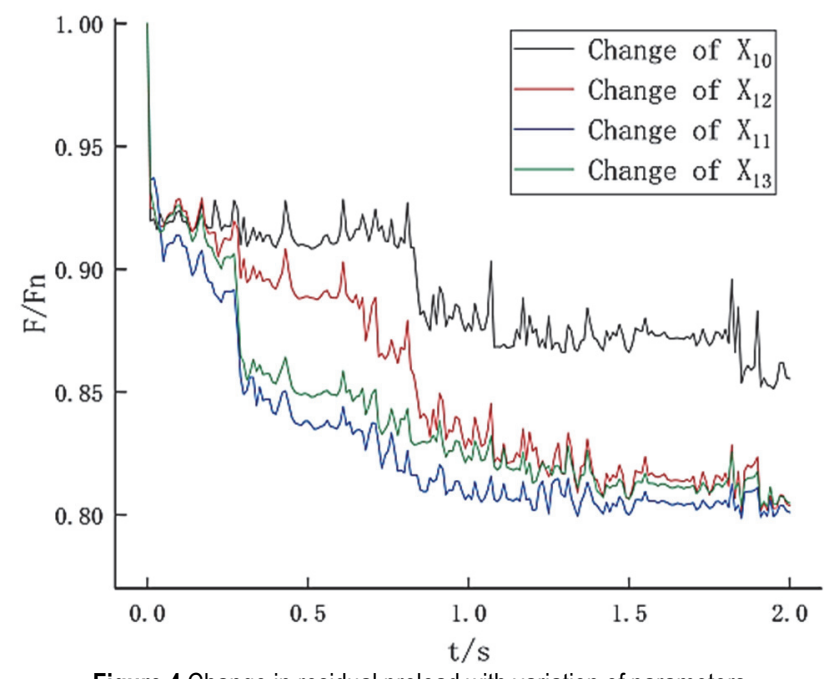

Figure 4 Change in residual preload with variation of parameters

\section{CONCLUSIONS}

1) The method for analyzing the correlation grades of influencing factors of bolted connections can be used to analyze multiple influencing factors at the same time, thus simplifying the analytical process.

2) The proposed method based on grey correlation degree analysis resulted in improvements in three key areas: dimensionless processing of factors, resolution coefficients, and weight assignment. The method can also be combined with the difference classification method for grading correlation degrees. A qualitative analysis was performed on factors of the same grade regardless of the magnitudes of correlation degree. This approach optimizes the selection of influencing factors in a targeted manner, avoids the adverse influence of variable correlation orders due to slight changes in differences, and achieves reasonable results.

3) The bolted connections on a third-rail current collector slide were taken as an example. It was found that to strengthen the reliability of bolted connections, it is necessary to first select a suitable bolt material and size, and then to optimize the friction conditions of various contact surfaces. Finally, the reliability of the proposed method was verified.

\section{Acknowledgments}

This work was supported by the Hunan Natural Science Foundation in China (2018JJ4059) and the scientific research fund of Hunan Provincial Education Department (19C0577).

\section{REFERENCES}

[1] Shao, J., Wang, T., Wang, Z. et al. (2019). Bolt Looseness Detection Using Pizoelectric Impedance Frequency Shift Method. China Mechanical Engineering, 30(12), 1395$1399+1408$.

[2] Gerbo, E. J., Thrall, A. P., \& Zoli, T. P. (2020). Service and Ultimate Behavior of Adjustable Bolted Steel Plate Connections. Journal of Structural Engineering, 146(7). https://doi.org/10.1061/(ASCE)ST.1943-541X.0002635

[3] Hou, S. (2015). Study on Mechanism of Loosening of Threaded connection. Doctoral Dissertation, Beijing: Beijing Institute of Technology. 
[4] Vinogradov, O. \& Huang, X. (1989). On a high frequency mechanism of self-loosening of fasteners. Proceedings of 12th ASME conference on mechanical vibration and noise, 131-137.

[5] Yang, L. \& Wang, L. (2020). The Fixing Structure Optimization Design on Bolted Connection. Missiles and Space Vehicles, (02), 49-53.

[6] Ren, H., Wang, D., Zhu, H. et al. (2020). Influence of Number of Shaft Flange Connection Bolts on Vibration. Ship Enginee, 42(03), 69-73.

[7] Wang, W., Xu, H., Ma, Y. et al. (2014). Self-loosening mechanism of bolted joints under vibration. Journal of Vibration and Shock, (22), 198-202. https://doi.org/10.1115/IMECE2014-37971

[8] Yang, G., Hong, J., Zhu, L., Li, B., Xiong, M., \& Wang, F. (2013). Three-dimensional Finite Element Analysis of the Mechanical Properties of Helical Thread Connection. Chinese Journal of Mechanical Engineering, 26(03), 564572. https://doi.org/10.3901/CJME.2013.03.564

[9] Junker, G. H. (1969). New criteria for self-loosening of fasteners under vibration. Sae Transactions, 78, 314-335. https://doi.org/10.4271/690055

[10] Yang, F., Li, Z., Zhang, D. et al. (2018). Experimental study on the transversal vibration of double-nut bolted joints of transmission towers. Journal of Vibration and Shock, 37(10), 164-171.

[11] Li, Z., Guan, Z., Zhang, J. et al. (2012). Applied Fracture Mechanics. Beijing: Beihang University Press.

[12] Wang, H., Jing, W., Zhao, G. et al. (2020). Fatigue Life Prediction for Hydraulic Support Foundation Based or Grey System Model GM $(1,1)$ Improved Miner Criterion. Journal of Shanghai Jiao Tong University, 54(01), 106-110.

[13] Wang, J., He, S., Wang, W. et al. (2018).Study on Wind Speed Prediction Based on Mixed Grey Theory. Acta Energiae Solaris Sinica, 39(12), 3544-3549.

\section{Contact information:}

\section{Qishui YAO}

School of Mechanical Engineering,

Hunan University of Technology,

Zhuzhou, Hunan Province, 412000, China

\section{Qiuwei YUAN}

School of Mechanical Engineering,

Hunan University of Technology,

Zhuzhou, Hunan Province, 412000, China

\section{Jianghong YU}

(Corresponding author)

School of Mechanical Engineering,

Hunan University of Technology,

Zhuzhou, Hunan Province, 412000, China

E-mail: hutyjh@hut.edu.cn

\section{Yaoyao DENG}

School of Mechanical Engineering,

Hunan University of Technology,

Zhuzhou, Hunan Province, 412000, China 